\title{
Targeted therapies for gastroesophageal cancers
}

\author{
Andrew Hsu, Rani Chudasama, Khaldoun Almhanna, Alexander Raufi \\ Division of Hematology/Oncology, The Warren Alpert Medical School of Brown University, Lifespan Cancer Institute, Rhode Island Hospital, \\ Providence, RI, USA \\ Contributions: (I) Conception and design: None; (II) Administrative support: None; (III) Provision of study materials or patients: None; (IV) \\ Collection and assembly of data: None; (V) Data analysis and interpretation: None; (VI) Manuscript writing: All authors; (VII) Final approval of \\ manuscript: All authors. \\ Correspondence to: Andrew Hsu, MD. Division of Hematology/Oncology, The Warren Alpert Medical School of Brown University, 593 Eddy St, \\ George Building, Providence, RI 02903, USA. Email: andrew_hsu1@brown.edu.
}

\begin{abstract}
Gastroesophageal cancers are some of the most common malignancies worldwide. A significant portion of patients are diagnosed with advanced or metastatic disease given the insidious nature of gastroesophageal cancers. In the instance where surgical resection for cure is no longer an option, the prognosis is poor and generally less than a year. Traditionally, standard front-line chemotherapy included two- to three-drug regimens with modest improvements in overall survival. Over the past two decades, with increased understanding of the biology of cancer, targeted therapies have been developed to stop the actions of molecules that are key in the growth and spread of cancer cells and have been successful in a number of cancers. In gastroesophageal cancer, these gains have been more modest with limited approval—trastuzumab being incorporated into front-line use in HER2-positive disease, and ramucirumab alone or in combination with paclitaxel becoming the preferred second-line regimen in progressive disease. However, with increased understanding of the biology of cancer, new and promising targeted therapies have emerged along with novel strategies in combining targeted therapies with traditional chemotherapy and immunotherapy. In this article, we will review the use of targeted therapies in the treatment of gastroesophageal cancer and touch upon future treatment strategies and therapeutics currently under investigation.
\end{abstract}

Keywords: Gastric cancer; esophageal cancer; gastroesophageal cancer; targeted therapy; trastuzumab; ramicurumab

Submitted Apr 02, 2020. Accepted for publication Jun 30, 2020.

doi: $10.21037 /$ atm-20-3265

View this article at: http://dx.doi.org/10.21037/atm-20-3265

\section{Introduction}

Gastric and esophageal cancers, collectively termed as gastroesophageal cancers, are among the leading causes of cancer death worldwide. Esophageal cancer is the eighteenth most common malignancy in the United States by incidence-17,650 new cases and $1.0 \%$ of all new cancers diagnosed in 2019. Furthermore, esophageal cancer accounted for $2.6 \%$ of cancer-related deaths in 2019 (1). Globally, esophageal cancer is the eighth most common malignancy and the sixth leading cause of cancerrelated death (2). There are two major histological subtypes of esophageal cancer: squamous cell carcinoma (SCC) and adenocarcinoma. SCC remains the most common histology worldwide; however, within the past half century, adenocarcinoma has become the most common subtype in the Western World and within the United States (3). This shift in histological subtype has been largely attributed to changes in lifestyle such as decreased rates of tobacco and alcohol use and increased rates of obesity and associated comorbidities such as Barrett's esophagus or gastroesophageal reflux disease (GERD) (4). For localized or resectable disease, the 5 -year survival is $19.9 \%$; however, the 5 -year survival for metastatic disease is $4.8 \%$ (1).

Although the incidence of gastric cancer in the United States is in a decline, it remains a significant health issue, 
with 27,510 new cases and 11,140 deaths in 2019. Globally, gastric cancer represents a substantial disease burden; the fifth most common malignancy and third leading cause of death from cancer (5). Its incidence worldwide varies depending on the geographical region, a high incidence in Asia, Latin American, Central and Eastern Europe, and a low incidence in Western Europe and North America. This dichotomy is multifactorial and decreasing prevalence of Helicobacter pylori infection likely plays a large role (6). The 5-year survival rates for localized and metastatic gastric cancer mirror those of esophageal cancer and are approximately $31.5 \%$ and $5.3 \%$, respectively (7).

Patients with early-stage gastroesophageal cancer may present with anorexia, dyspepsia, dysphagia, abdominal pain, or weight loss; however, approximately $40 \%$ of patients remain asymptomatic and are diagnosed late, with metastatic or unresectable disease $(1,7)$. In earlier stages, multimodality treatment is often required, with a combination of surgery and chemotherapy, with or without radiation. Multiple strategies exist on how to deliver neoadjuvant and adjuvant chemotherapy; however, no global consensus about the optimal strategy exists (8). With some notable exceptions, such as in the case of esophageal SCC, surgical resection often remains necessary to achieve a cure. In the setting of metastatic or unresectable disease, the prognosis remains poor and traditional standard-of-care (SOC) therapies offer limited impact on patient outcomes with median survival ranging between four months with best supportive care (BSC), to twelve months with the use of cytotoxic chemotherapy $(9,10)$.

Over the past few decades, our understanding of the molecular pathogenesis and biology of cancer has increased significantly. This has led to the development of novel targeted therapeutic strategies aimed at improving survival. Currently, targeted therapies are available in two forms: monoclonal antibodies (mAb) and small molecule inhibitors, the majority of which are tyrosine kinase inhibitors (TKIs) (Figure 1). In this review article we aim to summarize currently approved targeted therapies for the treatment of gastroesophageal cancer as well as highlight possible future treatments currently under investigation.

We present the following article in accordance with the Narrative Review reporting checklist (available at http:// dx.doi.org/10.21037/atm-20-3265).

\section{Epidermal growth factor receptor (EGFR)}

EGFR is a transmembrane receptor tyrosine kinase that normally regulates epithelial tissue development and homeostasis. It is a member of the erythroblastic oncogene B (ErbB) family of receptors and overexpressing this receptor or constitutive activation has been shown to increase proliferation, metastasis, and resistance to apoptosis (11). Aberrant expression has been linked to tumorigenesis in a variety of cancers (11). In gastroesophageal cancer, EGFR is overexpressed in $27-55 \%$ of cases and is associated with poor prognosis (12-14). Given the high rates of overexpression, EGFR is an attractive potential target for treatment of gastroesophageal cancer.

Cetuximab was initially approved in 2004 and became the first commercially available mAb targeting EGFR. It has been approved for the treatment of colorectal cancer, SCC of the head and neck, and NSCLC. Several trials have sought to determine its efficacy in gastroesophageal cancer with several phase II studies demonstrating improvements in median overall survival (mOS) in patients with metastatic gastric cancer supporting further exploration (15-18). The EXPAND trial sought to examine its use in the front-line setting for metastatic gastric and gastroesophageal junction (GEJ) adenocarcinoma. This phase III trial included 904 patients and compared the combination of cisplatin and capecitabine with or without cetuximab and demonstrated a trend towards inferior median progression free survival (mPFS) (4.4 vs. 5.6 months; HR 1.09; 95\% CI, 0.92-1.29; $\mathrm{P}=0.32$ ) and inferior mOS (9.4 vs. 10.7 months; HR 1.00; 95\% CI, 0.87-1.12; $\mathrm{P}=0.95)$. Toxicities were similar between the two arms except for an increased incidence of a rash with cetuximab use (19). Cetuximab has also been examined in the neoadjuvant setting in the phase II/III SCOPE-1 trial. Patients with resectable esophageal cancer were randomized to receive chemoradiotherapy (CRT) (capecitabine and cisplatin) alone or CRT with cetuximab. The trial was ended prematurely, prior to the phase III portion, because the trial met criteria for futility after demonstrating inferior mOS (22.1 vs. 25.4 months; HR 1.53; 95\% CI, 1.03-2.27; P=0.035) (20).

Another anti-EGFR mAb, panitumumab, has also been examined in gastroesophageal cancer. The REAL-3 trial examined this agent in conjunction with chemotherapy in untreated locally-advanced or metastatic gastroesophageal adenocarcinoma. This phase II/III trial randomized 503 patients to receive panitumumab with modified epirubicin, oxaliplatin, and capecitabine (EOX) versus placebo with EOX. The addition of panitumumab to modified EOX demonstrated inferior mOS when compared to EOX (8.8 vs. 11.3 months; HR 1.37; 95\% CI, 1.07 - 


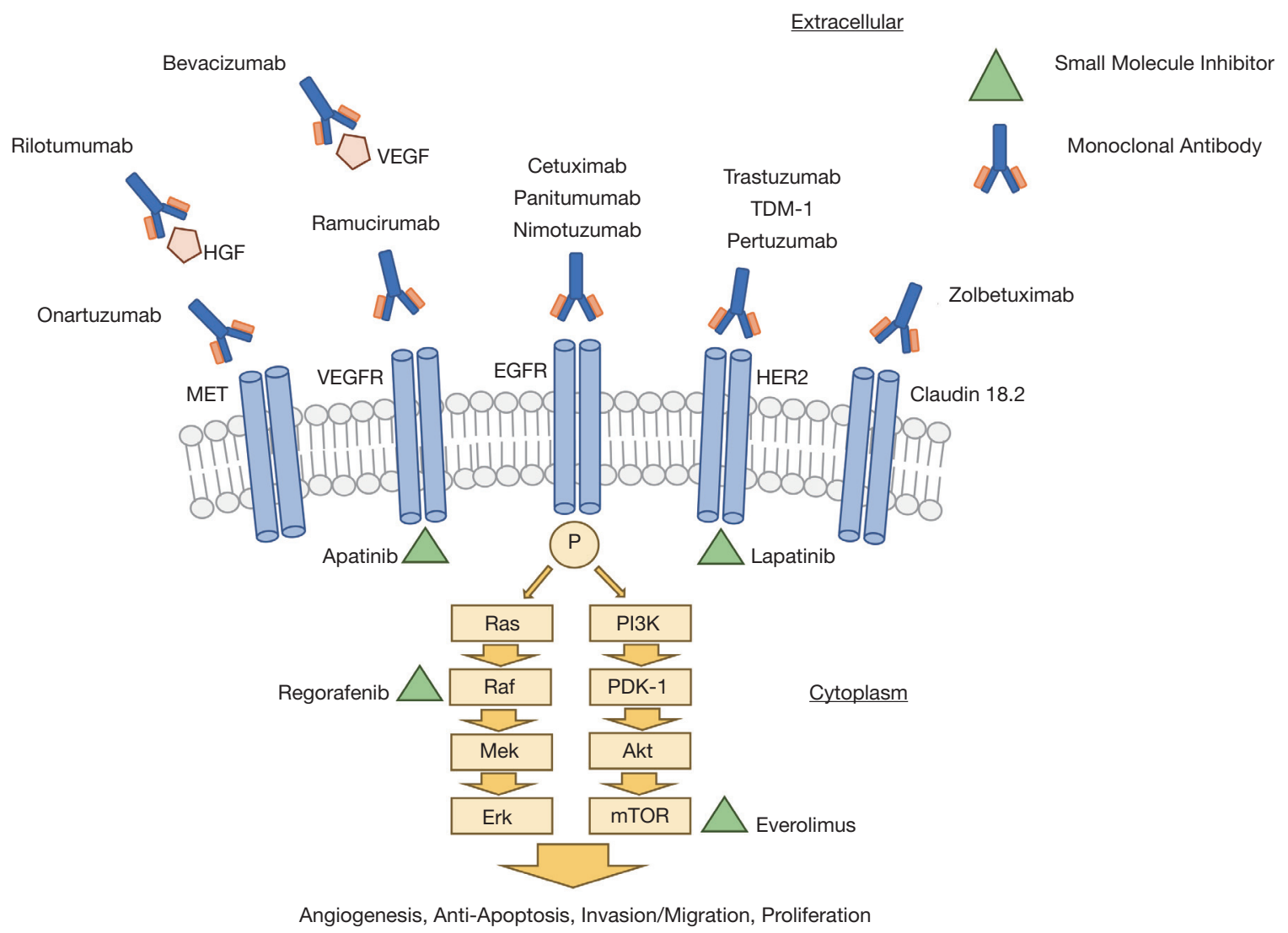

Figure 1 Monoclonal antibodies and tyrosine receptor kinase inhibitors and their targets in gastroesophageal cancer-mesenchymalepithelial transition (MET), hepatocyte growth factor (HGF), vascular endothelial growth factor (VEGF), epidermal growth factor receptor (EGFR), human epidermal growth factor 2 (HER2), claudin-18 isoform 2 (Claudin 18.2), rapidly accelerated fibrosarcoma (Raf), mammalian target of rapamycin (mTOR).

1.76; $\mathrm{P}=0.013)$. Furthermore, despite dose-reductions in oxaliplatin and capecitabine, toxicities were increased with panitumumab, particularly grade $3-4$ diarrhea (17\% vs. $11 \%)$, rash (11\% vs. $1 \%)$, and mucositis (5\% vs. $0 \%)(21)$. Panitumumab has also been examined in the neoadjuvant setting for resectable esophageal adenocarcinoma. Panitumumab was given in combination with docetaxel and cisplatin with concurrent radiotherapy in a phase II study with 70 patients and demonstrated a pathologic complete response (pCR) of $33 \%$ and near-pCR of $20.4 \%$; however, this was associated with significant toxicity as $48.5 \%$ had grade 4 toxicity with a $3.7 \%$ operative mortality (22). Given the significant toxicity, the authors argued that this regimen should not be further evaluated in a phase III trial.

A third anti-EGFR mAb, nimotuzumab, has been evaluated in combination with cisplatin and S-1 (CS). Both of these agents are approved for the treatment of metastatic gastroesophageal cancer in many Asian countries, as front- line treatment for unresectable or metastatic gastric cancer. In a phase II trial, 62 patients were randomized to receive nimotuzumab plus CS versus CS alone and unfortunately resulted in no improvement in the overall response rate (ORR) (54.8\% vs. 58.1\%; $\mathrm{P}=0.798)$, inferior mPFS (4.8 vs. 7.2 months; HR 2.136; 95\% CI, 1.19-3.83; $\mathrm{P}=0.011$ ), and inferior mOS (10.2 vs. 14.3 months; HR 1.776; 95\% CI, 0.97-3.25; $\mathrm{P}=0.062)(23)$.

As in NSCLC, the failure of mAbs to improve outcomes in patients prompted trials testing novel EGFR TKIs. Erlotinib and gefitinib are small molecule TKIs; the latter of which demonstrated activity with a signal for benefit in phase II trials $(24,25)$. In a phase III study, gefitinib monotherapy was compared to BSC in patients with metastatic esophageal cancer after failure of two lines of chemotherapy and one line of chemoradiotherapy. Gefitinib demonstrated a modest improvement in mPFS (1.57 vs. 1.17 months; HR 0.80; 95\% CI, 0.66-0.96; $\mathrm{P}=0.020$ ), 
but no improvement of mOS (3.73 vs. 3.67 months; HR 0.90; 95\% CI, 0.74-1.09; $\mathrm{P}=0.29)$ (26). Gefitinib was also examined in the neoadjuvant setting in the treatment of locally-advanced gastroesophageal cancer in combination with cisplatin and fluorouracil with concurrent radiotherapy followed by maintenance and showed no difference in locoregional control ( $77 \%$ vs. $76 \%$, respectively; $\mathrm{P}=0.006$ ) or distant metastatic control (40\% vs. 32\%, respectively; $\mathrm{P}=0.33$ ) (27). Furthermore, $48 \%$ of patients were intolerant of maintenance gefitinib secondary to increased rates of toxicity.

Despite the high prevalence of EGFR overexpression in gastroesophageal cancers, EGFR-targeted therapies have been largely disappointing when examined in large randomized trials. Table 1 summarizes the landmark trials without improvement in outcomes. Neither improvement in $\mathrm{mPFS}$ nor mOS has been demonstrated and except for use in a clinical trial these agents currently have no role in the treatment of gastroesophageal cancer.

\section{Human epidermal growth factor 2 (HER2)}

HER2 is a protein encoded by the ErbB2, and like EGFR, is a member of the ErbB family. Dimerization leads to downstream activation of signaling pathways which modulate gene expression driving cell cycle progression, proliferation, resistance to apoptosis, and tumorigenesis (39). Akin to breast cancer, HER2 has become an attractive target in the treatment of gastroesophageal cancer in which a large subset of cases exhibit HER2 amplification, resulting in HER2-receptor overexpression in up to $30 \%$ of cases (40).

Trastuzumab, a mAb targeting HER2, was approved in 2010 for front-line use in combination with chemotherapy for metastatic, HER2-positive gastric and GEJ adenocarcinoma based on the results of the ToGA trial (41). In this phase III trial, 584 patients were randomized to trastuzumab with a fluoropyrimidine and cisplatin versus a fluoropyrimidine with cisplatin only. HER2-positivity was defined as $3+$ on immunohistochemistry (IHC) or a HER2:CEP17 ratio of 2 or greater by fluorescence in situ hybridization (FISH). The addition of trastuzumab to chemotherapy led to an improvement in mPFS (6.7 vs. 5.5 months; HR 0.71; 95\% CI, 0.59-0.85; $\mathrm{P}=0.0002)$ and $\mathrm{mOS}$ (13.8 vs. 11.1 months; HR 0.74; 95\% CI, 0.60-0.91; $\mathrm{P}=0.0046)$. Furthermore, the trastuzumab arm demonstrated an improvement in the duration of response (6.9 vs. 4.8 months; HR 0.53; 95\% CI, 0.40-0.73; $\mathrm{P}<0.0001)$, ORR (47\% vs. $35 \% ; \mathrm{P}=0.0017)$, and a prolonged time to progression (TTP) (7.1 vs. 5.6 months; HR 0.70; 95\% CI, 0.58-0.85; P=0.0003) (41). Trastuzumab was generally well-tolerated without any overall difference in adverse effects. Post hoc analyses revealed that patients with high HER2-positive disease, defined as IHC $2+$ with FISH positivity or IHC $3+$, derived the most benefit from trastuzumab while an IHCs of 0 or $1+$ were unlikely to benefit regardless of FISH positivity (41).

The phase III HELOISE trial evaluated the efficacy of higher doses of trastuzumab for the treatment of metastatic gastroesophageal cancer. The rationale for this trial was the post hoc finding that patients in the ToGA trial with the lowest quartile of the trastuzumab serum trough concentration had a shorter mOS. It was theorized that higher doses of trastuzumab would lead to an increase in serum trough levels to improve mOS (42). Patients were randomized to receive the standard loading-dose of trastuzumab at $8 \mathrm{mg} / \mathrm{kg}$ followed by maintenance $6 \mathrm{mg} / \mathrm{kg}$ versus a dose-escalated maintenance dose of $10 \mathrm{mg} / \mathrm{kg}$. While serum concentrations were increased, this trial showed no improvement in mOS (10.6 vs. 12.5 months; HR 1.24; 95\% CI, 0.86-1.78; $\mathrm{P}=0.2401$ ) with comparable toxicity profiles (43). Other subsequent trials have sought to evaluate different fluoropyrimidine and platinum combinations with trastuzumab in an effort to reduce side effects (44-46). The results from these trials suggested that in combination with trastuzumab, fluorouracil or capecitabine, and cisplatin or oxaliplatin can be used interchangeably without significant reduction in efficacy.

In the last decade, the discovery of immune checkpoint inhibition has been considered a major breakthrough in the treatment of cancer; however, its success as a monotherapy in gastroesophageal cancer has been modest when compared to its use in other cancers. In an attempt to improve outcomes, immunotherapy is now being examined in combination with cytotoxic chemotherapies and targeted therapies. Janjigian et al. examined the use of trastuzumab in combination with pembrolizumab with a platinum and a fluoropyrimidine in 37 patients with metastatic gastric, esophageal, or GEJ cancer regardless of program cell death ligand 1 (PD-L1) expression. The primary endpoint was achieved in this phase II trial, with 26 of 37 patients $(70 \%)$ remaining progression free at 6 months (47). This promising data is being further examined in the phase III clinical trial, KEYNOTE-811 (48).

Given its success in metastatic HER2-positive disease, trastuzumab has been examined in both the neoadjuvant and adjuvant settings. Safran et al. examined the neoadjuvant use 
Table 1 Landmark trials with non-significant improvement or inferior outcomes

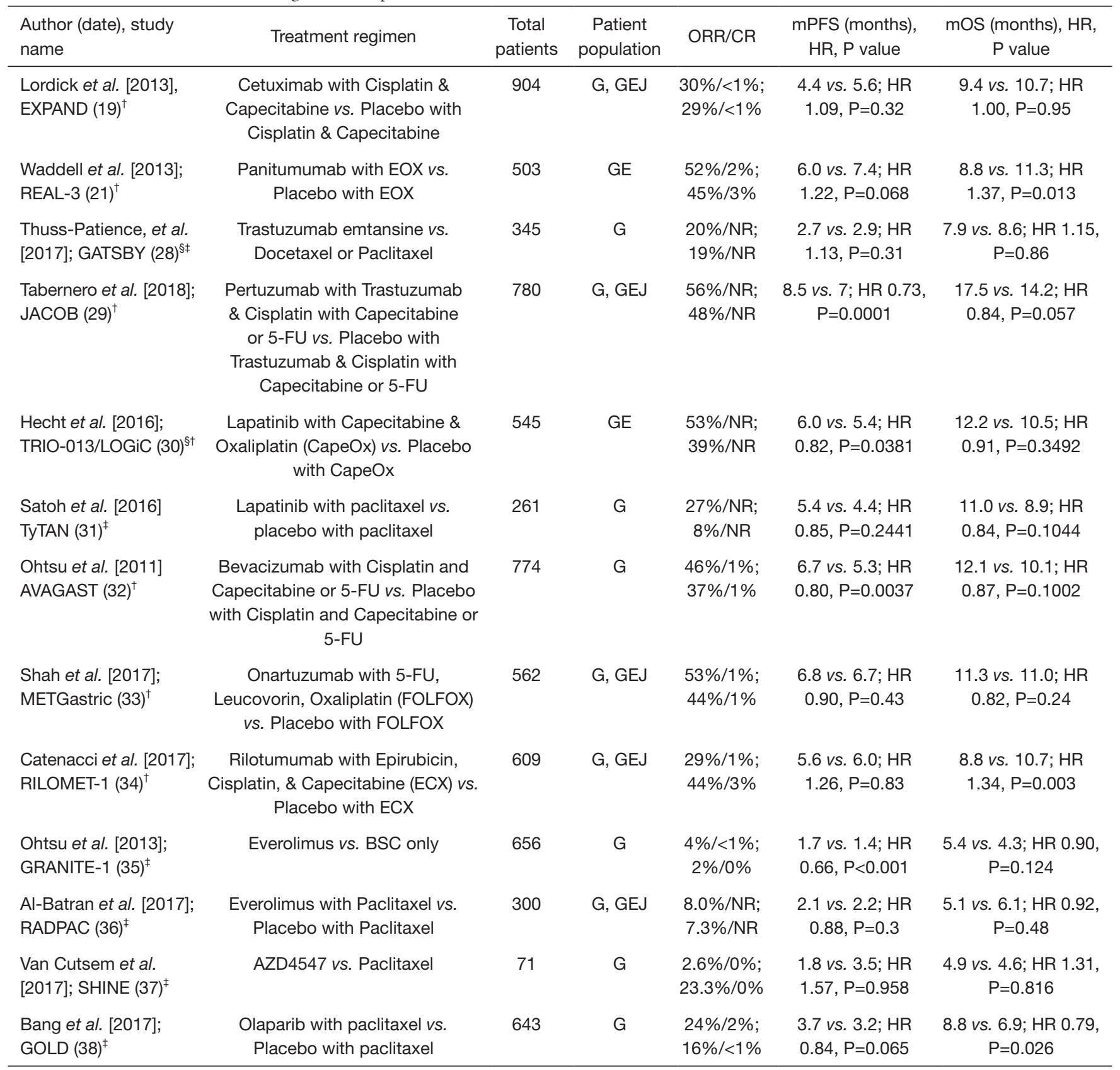

§, HER2-positive only; ${ }^{\dagger}$, first-line setting; ${ }^{\ddagger}$, second-line or further setting. NR, not reported; BSC, best supportive care; G, gastric, GEJ, gastroesophageal junction; GE, gastroesophageal.

of trastuzumab with paclitaxel, carboplatin and concurrent radiotherapy in locally-advanced HER2-positive esophageal adenocarcinoma. This phase III study enrolled 571 patients and found no improvement in mOS (38.5 vs. 38.9 months; HR 1.01; 95\% CI, 0.69-1.47) (49). The phase I TOXAG trial, trastuzumab combined with oxaliplatin, capecitabine and concurrent radiotherapy was given to HER2-positive gastric or GEJ adenocarcinoma patients who had undergone curative resection. This trial completed accrual in October 2019 and preliminary results showed that $90.3 \%$ of patients tolerated the combination regimen, but efficacy findings are pending (50). As in the MAGIC trial, several studies have 
attempted to incorporate trastuzumab into perioperative chemotherapy regimens. The NeoHX study combined trastuzumab with capecitabine and oxaliplatin (CapeOx) while HER-FLOT combined trastuzumab with docetaxel, oxaliplatin, 5-FU, and leucovorin (FLOT). These phase II trials demonstrated a pCR of $8.3 \%$ and $22.2 \%$, respectively, and has prompted ongoing phase III trials with similar treatment backbones (51-53).

Trastuzumab emtansine (T-DM1) is an antibodydrug conjugate combining trastuzumab with emtansine, a microtubule polymerization inhibitor. In the phase II/III GATSBY trial, T-DM1 was compared to taxane for use in the second-line setting for HER2-positive, unresectable, locally advanced, or metastatic gastric and GEJ adenocarcinoma. Of the 228 patients assigned to receive T-DM1, 173 (76\%) had previously received trastuzumab. The GATSBY trial failed to improve mOS (7.9 vs. 8.6 months; HR 1.15; 95\% CI, 0.87-1.51; $\mathrm{P}=0.86$ ) (28). In the phase I/II TRAX-HER2 trial, T-DM1 was compared to T-DM1 with capecitabine in HER2-positive locallyadvanced or metastatic gastric cancer after progressing on one or more lines of trastuzumab-based therapy and ultimately found no statistical difference in ORR (44.4\% vs. $36.3 \% ; \mathrm{P}=0.336$ ) (54). The role of $\mathrm{T}-\mathrm{DM} 1$ in patients who have progressed on trastuzumab is unclear and further investigation is needed.

Trastuzumab deruxtecan (DS-8201) is an antibodydrug conjugate combining trastuzumab with deruxtecan, a topoisomerase I inhibitor. In the phase II DESTINYGastric01 trial, 187 patients with previously treated HER2positive advanced gastric cancer were randomized at a 2:1 ratio to DS-8201 or physicians' choice of chemotherapy. DS-8201 demonstrated a higher ORR (51\% vs. 14\%, $\mathrm{P}<0.001)$ along with an improved mOS (12.5 vs. 8.4 months; HR 0.59; 95\% CI, 0.39-0.88; P=0.01). The most common adverse effects included myelosuppressive along with drug-induced interstitial lung disease or pneumonitis (55). Currently, the ongoing phase II trial, DESTINY-Gastric02, is examining the second-line use of DS-8201 after receiving first-line trastuzumab (56).

Pertuzumab, a mAb targeting a different epitope of HER2 than trastuzumab, was examined in the firstline setting in the JACOB trial. This phase III trial examined the addition of pertuzumab to trastuzumab with chemotherapy (i.e., a fluoropyrimidine, cisplatin) versus placebo with trastuzumab and chemotherapy in patients with metastatic, HER2-positive gastric or GEJ adenocarcinoma. This study demonstrated no significant improvement in mOS (17.5 vs. 14.2 months; HR 0.84; $95 \%$ CI, 0.71-1.00; $\mathrm{P}=0.057)$ (29). There are several phase II trials evaluating the use of pertuzumab in combination with trastuzumab in the neoadjuvant and perioperative setting such as the INNOVATE and Petrarca, respectively $(57,58)$. The final results of Petrarca were recently presented and demonstrated that the addition of pertuzumab and trastuzumab to FLOT leading to a significantly improved pCR $(35 \%$ vs. $12 \%, \mathrm{P}=0.02)$ and pathological lymph node negativity in HER2-positive esophagogastric adenocarcinoma (58). Other mAb targeting HER2 that are currently under investigation includes ZW25 and margetuximab $(59,60)$.

Lapatinib is a dual antagonist of both HER2 and EGFR and was studied as first-line therapy for metastatic gastroesophageal adenocarcinoma in the phase III LOGiC/ TRIO-013 trial which compared lapatinib with CapeOx to CapeOx alone. The addition of lapatinib led to a nonsignificant improvement in mOS (12.2 vs. 10.5 months; HR 0.91; 95\% CI, 0.73-1.12; $\mathrm{P}=0.3492)$ and mPFS (6.0 vs. 5.4 months; HR 0.82; 95\% CI, 0.68-1.00; $\mathrm{P}=0.0381$ ) (30). Lapatinib was also examined in the second-line setting in combination with paclitaxel versus paclitaxel alone in the phase III TyTAN trial. This trial enrolled 261 Asian patients with unresectable or metastatic gastric cancer. While the addition of lapatinib improved ORR (OR 3.85, $\mathrm{P}<0.001$ ), it failed to improve mOS (11.0 vs. 8.9 months; HR 0.84; 95\% CI, 0.64-1.11; $\mathrm{P}=0.1044)$, mPFS (5.4 vs. 4.4 months; HR $0.85 ; 95 \%$ CI, $0.63-1.13 ; \mathrm{P}=0.2441$ ), or TTP (5.5 vs. 4.4 months; HR 0.80; 95\% CI, 0.59-1.08; $\mathrm{P}=0.1321$ ) (31).

HER2 is one of the few successes in targeted therapies in the treatment of gastroesophageal cancers. The improvement in outcomes in the ToGA trial led to the incorporation of trastuzumab in the first-line setting when treating metastatic, HER2-positive disease (41). Table 2 summarized the landmark trials demonstrating improvement in outcome. Currently, there are ongoing trials examining the use of trastuzumab with and without pertuzumab in resectable, HER2-positive disease. Furthermore, immunotherapy is also being examined in combination with trastuzumab-based regimens in the treatment of gastroesophageal cancers (49).

\section{Vascular endothelial growth factors (VEGF)}

Angiogenesis is recognized as a key aspect of tumor growth, progression, and metastasis. VEGF, a key mediator of this process; binding several transmembrane tyrosine 
Table 2 Landmark trials with significant improvement in outcomes

\begin{tabular}{|c|c|c|c|c|c|c|}
\hline $\begin{array}{l}\text { Author (date), study } \\
\text { name }\end{array}$ & Treatment regimen & $\begin{array}{c}\text { Total } \\
\text { patients }\end{array}$ & $\begin{array}{c}\text { Patient } \\
\text { population }\end{array}$ & ORR/CR & $\begin{array}{l}\text { mPFS (months), } \\
\text { HR, P value }\end{array}$ & $\begin{array}{l}\text { mOS (months), HR, } \\
\text { P value }\end{array}$ \\
\hline $\begin{array}{l}\text { Bang et al. [2010], } \\
\text { ToGA }(41)^{\S \dagger}\end{array}$ & $\begin{array}{c}\text { Trastuzumab with cisplatin \& } \\
\text { Capecitabine or Fluorouracil (5-FU) }\end{array}$ & 584 & G, GEJ & $\begin{array}{l}47 \% / 5 \% \\
35 \% / 2 \%\end{array}$ & $\begin{array}{l}6.7 \text { vs. } 5.5 ; \mathrm{HR} 0.71 \\
\qquad \mathrm{P}=0.0002\end{array}$ & $\begin{array}{c}13.8 \text { vs. } 11.1 ; \mathrm{HR} \\
0.74, \mathrm{P}=0.0046\end{array}$ \\
\hline $\begin{array}{l}\text { Fuchs et al. [2014], } \\
\text { REGARD }(61)^{\ddagger}\end{array}$ & Ramucirumab vs. BSC only & 355 & G, GEJ & $\begin{array}{c}3 \% /<1 \% \\
3 \% / 0 \%\end{array}$ & $\begin{array}{c}2.1 \text { vs. } 1.3 ; \mathrm{HR} \\
0.483, \mathrm{P}<0.0001\end{array}$ & $\begin{array}{l}5.2 \text { vs. } 3.8 ; \mathrm{HR} \\
0.776, \mathrm{P}=0.47\end{array}$ \\
\hline $\begin{array}{l}\text { Pavlakis et al. [2016], } \\
\text { INTEGRATE }(63)^{\ddagger}\end{array}$ & Regorafenib vs. BSC only & 152 & G, GEJ & $\begin{array}{l}3 \% / N R ; \\
2 \% / N R\end{array}$ & $\begin{array}{l}2.6 \text { vs. } 0.9 ; \mathrm{HR} 0.40 \\
\mathrm{P}<0.001\end{array}$ & $\begin{array}{c}5.8 \text { vs. } 4.5 ; \mathrm{HR} 0.74, \\
\mathrm{P}=0.147\end{array}$ \\
\hline Li et al. [2016] (64) & Apatinib vs. BSC only & 267 & G, GEJ & $\begin{array}{l}3 \% / N R \\
0 \% / N R\end{array}$ & $\begin{array}{l}2.6 \text { vs. 1.8; HR } 0.44 \\
\quad P<0.001\end{array}$ & $\begin{array}{c}6.5 \text { vs. } 4.6 ; \mathrm{HR} 0.71, \\
\mathrm{P}=0.015\end{array}$ \\
\hline
\end{tabular}

NR, not reported; BSC, best supportive care; G, gastric; GEJ, gastroesophageal junction; GE, gastroesophageal; ${ }^{\S}$, HER2-positive only; ${ }^{\dagger}$, first-line setting; ${ }^{\ddagger}$, second-line or further setting.

kinase receptors (i.e., VEGFR-1 and VEGFR-2), and inducing angiogenesis. Hence, both VEGF and VEGFR family members have become attractive targets for cancer therapeutics.

Early studies with bevacizumab, a mAb targeting VEGF-A, showed promising preclinical activity which eventually led to the phase III AVAGAST trial where 774 treatment-naïve patients with inoperable, locally advanced or metastatic gastric or GEJ adenocarcinoma were randomized to fluoropyrimidine and cisplatin with or without bevacizumab (32). The trial demonstrated a small improvement in mPFS (6.7 vs. 5.3 months; HR $0.80 ; 95 \% \mathrm{CI}, 0.68-0.93 ; \mathrm{P}=0.0037)$ and a non-significant improvement in mOS (12.1 vs. 10.1 months; HR 0.87; 95\% CI, 0.73-1.03; P=0.1002) (32). Preplanned subgroup analysis of regional differences in outcomes revealed improved mOS in patients from North American and Latin American (11.5 vs. 6.8 months; HR 0.63; 95\% CI, 0.43-0.94); however, Asian patients saw no benefit which was attributed to regional differences in presentation and management of gastric cancr (32). Bevacizumab has also been evaluated for perioperative use for resectable gastroesophageal cancer in the MAGIC-B/ST03 trial. In this phase II/III trial, 1,063 patients were treated with perioperative epirubicin, cisplatin, and capecitabine (ECX) with or without bevacizumab. Again, there was no improvement in 3-year OS (48.1\% vs. 50.3\%, respectively;
HR 1.08; 95\% CI, 0.91-1.29; $\mathrm{P}=0.36$ ) with increased wound healing complications (12\% vs. $7 \%$ ) and higher incidence of grade 3 neutropenia in the bevacizumab arm (66).

Ramucirumab, an anti-VEGFR2 mAb, was examined in the first-line setting in the treatment of metastatic gastroesophageal cancer in the RAINFALL trial. In this phase III trial, 645 patients with metastatic, HER2-negative gastric or GEJ adenocarcinoma were randomized to receive ramucirumab plus a fluoropyrimidine and cisplatin or placebo plus a fluoropyrimidine and cisplatin. Ultimately, this trial failed to demonstrate significant improvement in mOS (11.2 vs. 10.7 months, HR 0.96; 95\% CI, 0.80-1.15; $\mathrm{P}=0.6757)$ and showed only a slight improvement in mPFS (5.7 vs. 5.4 months; HR 0.75; 95\% CI, 0.60-0.93; $\mathrm{P}=0.0106)$ (67). However, in the phase III REGARD trial, ramucirumab monotherapy was compared against BSC only in the second-line setting for patients with gastric or GEJ adenocarcinoma and demonstrated an improvement in mOS (5.2 vs. 3.8 months; HR 0.78; 95\% CI, 0.60-0.99; $\mathrm{P}=0.047)$ and mPFS (6.7 vs. 5.3 months; HR 0.80; 95\% CI, 0.68-0.93; $\mathrm{P}=0.037$ ) (61). Subsequently, the RAINBOW trial randomized 665 patients to receive ramucirumab plus paclitaxel or placebo plus paclitaxel. All patients had gastric or GEJ adenocarcinoma and had progressed on, or within 4 months, of first-line fluoropyrimidine-platinum based chemotherapy with or without an anthracycline. This phase III trial demonstrated a robust improvement 
in $\mathrm{mOS}$ (9.6 vs. 7.4 months; HR 0.81; 95\% CI, 0.68-0.96; $\mathrm{P}=0.0169$ ) (62). The results of the RAINBOW trial led to the approval of ramucirumab with paclitaxel in the secondline setting and is currently the preferred second-line regimen (62). Ramucirumab is currently being evaluated in the second-line setting in combination with irinotecan for patients previously treated with a fluoropyrimidine with or without a platinum and taxane in the RINDBeRG trial (68). Ramucirumab is also being evaluate in the second-line setting in combination with fluorouracil, leucovorin, and irinotecan (FOLFIRI) in the RIMIRIS trial. This phase II trial recently completed and demonstrated no improvement in mOS (6.8 vs. 7.6 months; $\mathrm{P}=0.77)$ and a non-significant improvement in PFS (4.6 vs. 3.6 months; $\mathrm{P}=0.12$ ) (69). RAMSES is a phase II/III trial that recently completed accrual in December 2019 that was examining the perioperative use of ramucirumab with FLOT and the results are pending (70).

Ziv-aflibercept is an antibody that binds both VEGF-1 and VEGF-2 which has been approved for use in colorectal cancer in conjunction with FOLFIRI (71). Given a theoretical advantage with dual VEGF binding, ziv-aflibercept was examined in combination with fluorouracil, leucovorin, and oxaliplatin (FOLFOX) in treatment-naïve patients with metastatic gastroesophageal adenocarcinoma but demonstrated no improvement in mOS, mPFS, or ORR (72).

Sunitinib and sorafenib are potent multitargeted TKIs, whose targets include VEGF, and is approved for use in many different cancers. The efficacy of both TKIs has been evaluated as monotherapy and in combination with chemotherapy in refractory disease without improvement in outcomes and has been associated with significant toxicities which has prohibited any subsequent phase III trials (73-78). Regorafenib is another multitargeted TKI whose targets include VEGF and is approved for use in gastrointestinal stromal tumor (GIST), hepatocellular carcinoma and colorectal cancer. It was evaluated for use in gastroesophageal cancer in the phase II INTEGRATE trial. A total of 152 patients with recurrent or metastatic gastric or GEJ adenocarcinoma refractory to one or more lines of chemotherapy were randomized at a two-to-one ratio to regorafenib. This trial found improvement in mPFS (2.6 vs. 0.9 months; HR 0.40; 95\% CI, 0.28-0.59; $\mathrm{P}<0.001)$ with a trend towards survival (5.8 vs. 4.5 months; HR 0.74; $\mathrm{P}=0.147$ ) (63). These results have led to the subsequent INTEGRATE II which is an ongoing phase III study examining the use of regorafenib in refractory disease (79). The phase Ib REGONIVO trial examined the use of regorafenib with nivolumab $3 \mathrm{mg} / \mathrm{kg}$ administered every two weeks in 50 patients with metastatic gastric or colorectal cancer who had received two or more lines of therapy. In gastric cancer, mPFS was 5.6 months $(95 \%$ CI, 2.7-10.4 months) and mOS was 12.3 months (95\% CI, 5.3-not reached). The most common grade 3 or more treatment-related adverse effect was rash $(12 \%)$, proteinuria (12\%), and palmar-plantar erythrodysesthesia (10\%) 80). REPEAT, a phase Ib trial examining the use of regorafenib with paclitaxel in the second-line setting, completed accrual in October 2019 and the results are pending (81).

Apatinib, a TKI targeting VEGFR-2, has been compared to BSC only in the third-line setting in patients with chemotherapy-refractory disease. In this phase II study, 144 Chinese patients were randomized to receive BSC only (group A), apatinib $850 \mathrm{mg}$ daily (group B), or apatinib $425 \mathrm{mg}$ twice daily (group $\mathrm{C}$ ) and demonstrated improvement in mOS in group B (2.50 vs. 4.83 months; HR 0.37; 95\% CI, $0.22-0.62 ; \mathrm{P}<0.001)$ and in group $\mathrm{C}(2.50$ vs. 4.27 months; HR 0.41; 95\% CI, 0.24-0.72; P=0.0017). Furthermore, there was an improvement in $\mathrm{mPFS}$ in both groups $(1.40$ vs. 3.67 vs. 3.20 months; HR 0.34; 95\% CI, 0.18-0.64; $\mathrm{P}<0.001)$. Grade 3 hand-foot syndrome (HFS) occurred more often in the apatinib arm (4\% and $13 \%$ in group B and $\mathrm{C}$, respectively) as well as grade 3 hypertension ( $9 \%$ and $11 \%$ in group B and C, respectively) (82). Subsequently, a phase III trial examined apatinib $850 \mathrm{mg}$ daily against BSC only in the third-line setting in 267 patients with chemotherapy-refractory gastric and GEJ adenocarcinoma and demonstrated improved mOS (6.5 vs. 4.6 months; HR $0.71 ; 95 \% \mathrm{CI}, 0.54-0.94 ; \mathrm{P}=0.015)$ and mPFS [2.6 vs. 1.8 months (HR 0.44; 95\% CI, 0.33-0.59; $\mathrm{P}<0.001$ )]. Like the prior phase II, grade 3 HFS ( $8.5 \%$ vs. $0 \%)$ and hypertension $(4.5 \%$ vs. $0 \%)$ occurred more often with apatinib (64). While these results are promising, there is little experience with apatinib outside of Asia. The phase III ANGEL study examined the use of apatinib in 460 who had failed at least two lines of prior therapy. This global study randomized patients to receive apatinib or placebo. In the third- and fourth-line setting, apatinib did not demonstrate any improvement in mOS (5.8 vs. 5.1 months; $\mathrm{P}=0.485$ ) but did demonstrate improvement in mPFS (2.8 vs. 1.8 months; $\mathrm{P}<0.0001)$. In fourth-line and beyond use, there was an improvement in mOS (6.3 vs. 4.7 months; $\mathrm{P}=0.0195)$ and mPFS (3.5 vs. 1.7 months; $\mathrm{P}<0.0001)$ (83).

The use of VEGF/VEGFR inhibitors has shown promise, with ramucirumab with paclitaxel now being incorporated into the treatment paradigm as the SOC 
second-line regimen in gastroesophageal cancer. Furthermore, it is currently being explored in the perioperative setting $(62,69)$. Other agents such as regorafenib and apatinib also have potential and are currently undergoing further evaluation (79).

\section{Claudin}

Claudins are a family of proteins involved in tight cell junctions which control the influx of molecules between cells forming epithelium and is highly expressed in differentiated epithelial cells of the gastric mucosa. Isoform 2 of claudin-18 (CLDN18.2) has been implicated in tumor development and progression and is present in approximately $50-70 \%$ of gastroesophageal tumors. This has spurred interest to develop mAbs targeting CLDN18.2 (84).

Zolbetuximab, also known as Claudiximab, is a $\mathrm{mAb}$ targeting CLDN18.2 which has been evaluated in the front-line setting in combination with EOX for advanced or recurrent, HER2-negative, CLDN18.2 expressing (defined as greater than $2+$ staining with anti-CLDN18 antibodies) gastroesophageal cancer in the FAST trial. This phase II trial demonstrated an improved mOS (13.0 vs. 8.4 months; HR 0.56; 95\% CI, 0.40-0.67; $\mathrm{P}=0.0008)$ and $\mathrm{mPFS}$ (7.5 vs. 5.3 months; HR 0.44; 95\% CI, 0.29-0.67; $\mathrm{P}<0.0005)$ (65). This convincing data served as the basis for the ongoing phase III SPOTLIGHT trial which is examining the role of zolbetuximab with FOLFOX versus FOLFOX alone in the front-line setting for patients with advanced or metastatic, HER2-negative, CLDN 18.2-expressing gastroesophageal cancer (85). The results are much anticipated as it will help determine what role CLDN18.2 inhibition will play in the treatment of gastroesophageal cancer.

\section{Mesenchymal-epithelial transition (MET)}

The MET oncogene encodes the protein c-Met, also called tyrosine-protein kinase Met or hepatocyte growth factor receptor (HGFR), which plays an essential role in embryonic development, organogenesis, as well as tissue repair and regeneration. However, in gastroesophageal cancer, overexpression of MET promotes cell proliferation, survival, and metastasis and is associated with a poor prognosis and more aggressive phenotype (86).

Onartuzumab is a mAb that binds to the extracellular domain of MET which prevents binding to its ligand, HGF, and thus preventing intracellular signaling. In the phase III METGastric trial, treatment-naïve patients with metastatic, HER2-negative, MET-positive (membranous and cytoplasmic staining of $1+$ or greater on IHC) gastric or GEJ adenocarcinoma were randomized to treatment with FOLFOX versus FOLFOX plus onartuzumab. Unfortunately, the addition of onartuzumab with FOLFOX did not improve mOS or mPFS (33).

Rilotumumab is a mAb that selectively targets HGF and blocks downstream cell proliferation, migration, and survival pathways. A phase Ib/II study compared rilotumumab versus placebo in combination with ECX for front-line use in patients with locally-advanced or metastatic gastroesophageal cancers and demonstrated improved mPFS and mOS independent of MET status. Of note, in a subgroup analysis of patients with MET-positive (at least $25 \%$ of tumor cells with membrane staining of $1+$ or greater intensity by IHC) tumors were found to have longer mPFS and mOS (87). These results led to RILOMET-1, a phase III study that assessed rilotumumab with ECX versus placebo with ECX for front-line use in patients with advanced MET-positive gastric or GEJ adenocarcinoma. Disappointingly, the study failed to demonstrate an improvement in mOS (8.8 vs. 10.7 months; $\mathrm{P}=0.003$ ) or mPFS (5.6 vs. 6.0 months; $\mathrm{P}=0.016$ ) and the study treatment was stopped early after an independent review found a higher number of deaths and toxicity in the rilotumumab arm (34). In both studies, the authors noted that MET overexpression on IHC inadequately selected the optimal patient population. Other explanations for the negative results were that targeting of MET signaling did not fundamentally affect tumor behavior or cancer outcomes and that alternative targeting in the MET pathway may be more fruitful. Given these findings, a role for METtargeted therapy in the treatment of gastroesophageal cancer remains undetermined.

\section{Mammalian target of rapamycin (mTOR)}

Phosphatidylinositol 3-kinase (PI3K) and mTOR are activated in $30 \%$ and $60 \%$ of gastric cancers, respectively (88). Dysregulation of the mTOR pathway is associated with chemotherapy-resistance and decreased survival (89). Everolimus, an oral mTOR inhibitor, has been shown to inhibit downstream signaling molecules, cell proliferation, tumor growth and vascularization and has shown clinical benefit and tolerability in various cancers. A phase II study evaluated everolimus in patients with advanced gastric cancer after progression on one or two lines of chemotherapy and showed a disease control rate of $54.7 \%$, mPFS of 2.7 months, and mOS of 10.1 months; however, 
no complete or partial responses were obtained (90). Everolimus has also been studied as a monotherapy and in combination with paclitaxel after progression on firstline treatment in the phase III GRANITE-1 and RADPAC studies, respectively. In both studies, everolimus was examined in patients with advanced gastric cancer who had progressed after one or more lines of therapy and failed to demonstrated improvement in $\mathrm{mPFS}$ or mOS and was associated with increased toxicity when compared to paclitaxel alone $(35,36)$. It is felt that the identification of a biomarker for various patient subpopulations with gastric cancer would help define and predict who would derive the most benefit from everolimus—an ongoing biomarker analysis of patients in GRANITE-1 is eagerly anticipated to help answer this question (35).

\section{Fibroblast-growth factor receptor (FGFR)}

The FGFR is a family of heparin-binding growth factor receptors that promote angiogenesis, cell proliferation, survival, migration and invasion, thus representing another potential therapeutic target. AZD4547 is a selective FGFR1-3 inhibitor which has been evaluated in the second-line setting in patients with advanced gastric adenocarcinoma displaying FGFR-2 polysomy or gene amplification by FISH. In the phase II Shine study, 71 patients were randomized 3:2 (FGFR2 gene amplification) or 1:1 (FGFR2 polysomy) to receive AZD4547 or paclitaxel. This study failed to meet its primary endpoint by demonstrating a trend towards inferior mPFS (1.8 vs. 3.5 months; HR 1.57; $\mathrm{P}=0.9581)$ and noted marked intratumor heterogeneity of FGFR2 amplification and poor concordance with amplification and polysomy (37). Currently, the role of FGFR inhibitors in the treatment of gastroesophageal cancer is unclear with further investigation ongoing.

\section{Poly(ADP-Ribose) Polymerase (PARP)}

Olaparib is an oral PARP inhibitor that inactivates PARP upon its binding to single-strand DNA breaks. This effectively prevents repair and generates DNA replication blocks leading to double-strand DNA breaks. Olaparib has shown clinical benefit in targeting tumors with deficiencies in double-strand DNA break repair such as such as those caused by homologous recombination repair deficiencies, for example in patients with BRCA 1/2 mutations $(91,92)$. In a phase II trial, involving Asian patients with advanced gastric cancer, olaparib combined with paclitaxel showed an improvement in mOS (13.1 vs. 8.3 months; HR 0.56; 80\% CI, 0.41-0.75; $\mathrm{P}=0.005)$ when compared to paclitaxel alone in the second-line setting. Half of the control and study arms were determined to have low ataxia telangiectasia mutated (ATM) levels on IHC and this population had a greater improvement in mOS $(\mathrm{P}=0.002)$ (93). Subsequently, the randomized phase III GOLD trial was conducted to determine efficacy of olaparib with paclitaxel in the second-line setting of Asian patients with advanced, ATMnegative gastric cancer. Unfortunately, this trial did not demonstrate any significant improvement in mOS (8.8 vs. 6.9 months; HR 0.79; 97.5\% CI, 0.63-1.00; $\mathrm{P}=0.026$ ) (38). PARP inhibition has been promising in other cancers; however, its role in treatment of gastroesophageal cancer is undetermined, requiring further studies to evaluate higher dosing, its use in BRCA-mutated populations, or patients previously treated with platinum-containing regimens.

\section{Conclusions}

While the use of targeted therapies have been successful in the treatment of cancers, making their way into frontline use in treatment paradigms for breast, colorectal, kidney, lung, melanoma, prostate, thyroid and certain hematological cancers, gains in gastroesophageal cancer have been more modest with few instances of success. Trastuzumab has found a role in combination with chemotherapy for front-line use in HER2-positive disease while ramucirumab with paclitaxel is the preferred secondline regimen in progressive disease. These targeted therapies are currently being evaluated for use in other settings-resectable disease, first- or second-line in combination with other chemotherapeutics, targeted therapies such as pertuzumab, or immunotherapies. Other agents, such as apatinib, regorafenib and zolbetuximab have shown promising phase II results and are currently being evaluated in larger randomized trials in hopes of further improving outcomes.

\section{Acknowledgments}

Funding: None.

\section{Footnote}

Provenance and Peer Review: This article was commissioned by the Guest Editor (Dr. Khaldoun Almhanna) for the series "Gastroesophageal Cancer 2020" published in Annals 
of Translational Medicine. The article was sent for external peer review organized by the Guest Editor and the editorial office.

Reporting Checklist: The authors have completed the Narrative Review reporting checklist. Available at http:// dx.doi.org/10.21037/atm-20-3265

Conflicts of Interest: All authors have completed the ICMJE uniform disclosure form (available at http://dx.doi. org/10.21037/atm-20-3265). The series "Gastroesophageal Cancer 2020" was commissioned by the editorial office without any funding or sponsorship. KA served as the unpaid Guest Editor of the series and serves as an unpaid editorial board member of Annals of Translational Medicine from Nov 2019 to Oct 2021. KA reports personal fees from Merck, outside the submitted work. The authors have no other conflicts of interest to declare.

Ethical Statement: The authors are accountable for all aspects of the work in ensuring that questions related to the accuracy or integrity of any part of the work are appropriately investigated and resolved.

Open Access Statement: This is an Open Access article distributed in accordance with the Creative Commons Attribution-NonCommercial-NoDerivs 4.0 International License (CC BY-NC-ND 4.0), which permits the noncommercial replication and distribution of the article with the strict proviso that no changes or edits are made and the original work is properly cited (including links to both the formal publication through the relevant DOI and the license). See: https://creativecommons.org/licenses/by-nc-nd/4.0/.

\section{References}

1. SEER Cancer Stat Facts; Esophageal Cancer Bethesda, MD: National Cancer Institute (NCI); 2019. Available online: https://seer.cancer.gov/statfacts/html/esoph.html

2. Fitzmaurice C, Allen C, Barber RM, et al. Global, Regional, and National Cancer Incidence, Mortality, Years of Life Lost, Years Lived With Disability, and DisabilityAdjusted Life-years for 32 Cancer Groups, 1990 to 2015: A Systematic Analysis for the Global Burden of Disease Study. JAMA Oncol 2017;3:524-48.

3. Islami F, Fedirko V, Tramacere I, et al. Alcohol drinking and esophageal squamous cell carcinoma with focus on light-drinkers and never-smokers: a systematic review and meta-analysis. Int J Cancer 2011;129:2473-84.

4. Engel LS, Chow WH, Vaughan TL, et al. Population attributable risks of esophageal and gastric cancers. J Natl Cancer Inst 2003;95:1404-13.

5. Ferro A, Peleteiro B, Malvezzi M, et al. Worldwide trends in gastric cancer mortality (1980-2011), with predictions to 2015, and incidence by subtype. Eur J Cancer 2014;50:1330-44.

6. Global Burden of Disease Cancer Collaboration; Fitzmaurice C, Allen C, et al. Global, Regional, and National Cancer Incidence, Mortality, Years of Life Lost, Years Lived With Disability, and Disability-Adjusted Lifeyears for 32 Cancer Groups, 1990 to 2015: A Systematic Analysis for the Global Burden of Disease Study. JAMA Oncol 2017;3:524-48.

7. SEER Cancer Stat Facts: Stomach Cancer Bethesda, MD: National Cancer Institute (NCI); 2019. Available online: https://seer.cancer.gov/statfacts/html/stomach.html

8. Lutz MP, Zalcberg JR, Ducreux M, et al. Highlights of the EORTC St. Gallen international expert consensus on the primary therapy of gastric, gastroesophageal and oesophageal cancer-differential treatment strategies for subtypes of early gastroesophageal cancer. Eur J Cancer 2012;48:2941-53.

9. Wagner AD, Unverzagt S, Grothe W, et al. Chemotherapy for advanced gastric cancer. Cochrane Database Syst Rev 2010;3:CD004064.

10. Glimelius B, Ekström K, Hoff man K, et al. Randomized comparison between chemotherapy plus best supportive care with best supportive care in advanced gastric cancer. Ann Oncol 1997;8:163-8.

11. Messa C, Russo F, Caruso MG et al. EGF, TGF-alpha, and EGFR in human colorectal adenocarcinoma. Acta Oncol 1998;37:285-9.

12. Tew WP, Kelsen DP, Ilson DH. Targeted therapies for esophageal cancer. Oncologist 2005;10:590-601.

13. Kim MA, Lee HS, Lee HE, et al. EGFR in gastric carcinomas: prognostic significance of protein overexpression and high gene copy number. Histopathology 2008;52:738-46.

14. Kopp R, Ruge M, Rothbauer E, et al. Impact of epidermal growth factor (EGF) radioreceptor analysis on longterm survival of gastric cancer patients. Anticancer Res 2002;22:1161-7.

15. Han SW, Oh DY, Im SA, et al. Phase II study and biomarker analysis of cetuximab combined with modified FOLFOX6 in advanced gastric cancer. Br J Cancer 2009;100:298-304. 
16. Moehler M, Muel ler A, Trarbach T, et al, and the German Arbeitsgemeinschaft Internistische Onkologie. Cetuximab with irinotecan, folinic acid and 5-fluorouracil as firstline treatment in advanced gastroesophageal cancer: a prospective multi-center biomarker-oriented phase II study. Ann Oncol 2011;22:1358-66.

17. Lordick F, Lube RB, Lorenzen S, et al. Cetuximab plus oxaliplatin/leucovorin/5-fluorouracil in firstline metastatic gastric cancer: a phase II study of the Arbeitsgemeinschaft Internistische Onkologie (AIO). Br J Cancer 2010;102:500-5.

18. Woll E, Greil R, Eisterer W, et al. Oxaliplatin, irinotecan and cetuximab in advanced gastric cancer. A multicenter phase II trial (Gastric-2) of the Arbeitsgemeinschaft Medikamentose Tumortherapie (AGMT). Anticancer Res 2011;31:4439-43.

19. Lordick F, Kang YK, Chung HC, et al. Capecitabine and cisplatin with or without cetuximab for patients with previously untreated advanced gastric cancer (EXPAND): a randomised, open-label phase 3 trial. Lancet Oncol 2013;14:490-9.

20. Crosby T, Hurt CN, Falk S, et al. Chemoradiotherapy with or without cetuximab in patients with oesophageal cancer (SCOPE1): a multicentre, phase 2/3 randomised trial. Lancet Oncol 2013;14:627-37.

21. Waddell T, Chau I, Cunningham D, et al. Epirubicin, oxaliplatin, and capecitabine with or without panitumumab for patients with previously untreated advanced oesophagogastric cancer (REAL3): a randomised, openlabel phase 3 trial. Lancet Oncol 2013;14:481-9.

22. Lockhart AC, Reed CE, Decker PA, et al. Phase II study of neoadjuvant therapy with docetaxel, cisplatin, panitumumab, and radiation therapy followed by surgery in patients with locally advanced adenocarcinoma of the distal esophagus (ACOSOG Z4051). Ann Oncol 2014;25:1039-44.

23. Du F, Zheng Z, Shi S, et al. S-1 and cisplatin with or without nimotuzumab for patients with untreated unresectable or metastatic gastric cancer: a randomized, open-label phase 2 trial. Medicine (Baltimore) 2015;94:e958.

24. Dragovich T, McCoy S, Fenoglio-Preiser CM et al. Phase II trial of erlotinib in gastroesophageal junction and gastric adenocarcinomas: SWOG 0127. J Clin Oncol 2006;24:4922-7.

25. Ferry DR, Anderson M, Beddard K, et al. A phase II study of gefitinib monotherapy in advanced esophageal adenocarcinoma: evidence of gene expression, cellular, and clinical response. Clin Cancer Res 2007;13:5869-75.

26. Dutton SJ, Ferry DR, Blazeby JM, et al. Gefitinib for oesophageal cancer progressing after chemotherapy (COG): a phase 3 , multicentre, double-blind, placebo-controlled randomised trial. Lancet Oncol 2014;15:894-904.

27. Rodriguez CP, Adelstein DJ, Rice TW, et al. A phase II study of perioperative concurrent chemotherapy, gefitinib, and hyperfractionated radiation followed by maintenance gefitinib in locoregionally advanced esophagus and gastroesophageal junction cancer. J Thorac Oncol 2010;5:229-35.

28. Thuss-Patience PC, Shah MA, Ohtsu A, et al. Trastuzumab emtansine versus taxane use for previously treated HER2positive locally advanced or metastatic gastric or gastrooesophageal junction adenocarcinoma (GATSBY): an international randomised, open-label, adaptive, phase 2/3 study. Lancet Oncol 2017;18:640-53.

29. Tabernero J, Hoff PM, Shen L, et al. Pertuzumab plus trastuzumab and chemotherapy for HER2-positive metastatic gastric or gastro-oesophageal junction cancer (JACOB): final analysis of a double-blind, randomised, placebo-controlled phase 3 study. Lancet Oncol 2018;19:1372-84.

30. Hecht JR, Bang YJ, Qin SK, et al. Lapatinib in Combination With Capecitabine Plus Oxaliplatin in Human Epidermal Growth Factor Receptor 2Positive Advanced or Metastatic Gastric, Esophageal, or Gastroesophageal Adenocarcinoma: TRIO-013/ LOGiC-A Randomized Phase III Trial. J Clin Oncol 2016;34:443-51.

31. Satoh T, Xu RH, Chung HC, et al. Lapatinib Plus Paclitaxel Versus Paclitaxel Alone in the Second-Line Treatment of HER2-Amplified Advanced Gastric Cancer in Asian Populations: TyTAN-A Randomized, Phase III Study. J Clin Oncol 2014;32:2039-49.

32. Ohtsu A, Shah MA, Van Cutsem E, et al. Bevacizumab in combination with chemotherapy as first-line therapy in advanced gastric cancer: a randomized, doubleblind, placebo-controlled phase III study. J Clin Oncol 2011;29:3968-76.

33. Shah MA, Bang YJ, Lordick F, et al. Effect of Fluorouracil, Leucovorin, and Oxaliplatin With or Without Onartuzumab in HER2-Negative, MET-Positive Gastroesophageal Adenocarcinoma: The METGastric Randomized Clinical Trial. JAMA Oncol 2017;3:620.

34. Catenacci DVT, Tebbutt NC, Davidenko I, et al. Rilotumumab plus epirubicin, cisplatin, and capecitabine as first-line therapy in advanced MET-positive gastric 
or gastro-oesophageal junction cancer (RILOMET-1): a randomised, double-blind, placebo-controlled, phase 3 trial. Lancet Oncol 2017;18:1467-82.

35. Ohtsu A, Ajani JA, Bai YX, et al. Everolimus for Previously Treated Advanced Gastric Cancer: Results of the Randomized, Double-Blind, Phase III GRANITE-1 Study. J Clin Oncol 2013;31:3935-43.

36. Al-Batran SE, Riera-Knorrenschild J, Pauligk C, et al. A randomized, double-blind, multicenter phase III study evaluating paclitaxel with and without RAD001 in patients with gastric cancer who have progressed after therapy with a fluoropyrimidine/platinum-containing regimen (RADPAC). J Clin Oncol 2017;35:4027.

37. Van Cutsem E, Bang YJ, Mansoor W, et al. A randomized, open-label study of the efficacy and safety of AZD4547 monotherapy versus paclitaxel for the treatment of advanced gastric adenocarcinoma with FGFR2 polysomy or gene amplification. Ann Oncol 2017;28:1316-24.

38. Bang YJ, Xu RH, Chin $\mathrm{K}$, et al. Olaparib in combination with paclitaxel in patients with advanced gastric cancer who have progressed following first-line therapy (GOLD): a double-blind, randomised, placebo-controlled, phase 3 trial. Lancet Oncol 2017;18:1637-51.

39. Baselga J, Swain SM. Novel anticancer targets: revisiting ERBB2 and discovering ERBB3. Nat Rev Cancer 2009;9:463-75.

40. Kim KC, Koh YW, Chang HM, et al. Evaluation of HER2 protein expression in gastric carcinomas: comparative analysis of 1,414 cases of whole-tissue sections and 595 cases of tissue microarrays. Ann Surg Oncol 2011;18:2833-40.

41. Bang YJ, Van Cutsem E, Feyereislova A, et al. Trastuzumab in combination with chemotherapy versus chemotherapy alone for treatment of HER2-positive advanced gastric or gastro-oesophageal junction cancer (ToGA): a phase 3, open-label, randomised controlled trial. Lancet 2010;376:687-97.

42. Cosson VF, Ng VW, Lehle M, et al: Population pharmacokinetics and exposure-response analyses of trastuzumab in patients with advanced gastric or gastroesophageal junction cancer. Cancer Chemother Pharmacol 2014;73:737-47.

43. Shah MA, Xu RH, Bang YJ, et al. HELOISE: Phase IIIb Randomized Multicenter Study Comparing Standard-ofCare and Higher-Dose Trastuzumab Regimens Combined With Chemotherapy as First-Line Therapy in Patients With Human Epidermal Growth Factor Receptor 2Positive Metastatic Gastric or Gastroesophageal Junction
Adenocarcinoma. J Clin Oncol 2017;35:2558-67.

44. Cunningham D, Starling N, Rao S, et al. Capecitabine and oxaliplatin for advanced esophagogastric cancer. N Engl J Med 2008;358:36-46.

45. Ryu MH, Yoo C, Kim JG, et al. Multicenter phase II study of trastuzumab in combination with capecitabine and oxaliplatin for advanced gastric cancer. Eur J Cancer 2015;51:482-8.

46. Soularue É, Cohen R, Tournigand C, et al. Efficacy and safety of trastuzumab in combination with oxaliplatin and fluorouracil-based chemotherapy for patients with HER2positive metastatic gastric and gastro-oesophageal junction adenocarcinoma patients: a retrospective study. Bull Cancer 2015;102:324-31.

47. Janjigian YY, Maron SB, Chatila WK, et al. First-line pembrolizumab and trastuzumab in HER2-positive oesophageal, gastric, or gastro-oesophageal junction cancer: an open-label, single-arm, phase 2 trial. Lancet Oncol 2020;21:821-31.

48. Pembrolizumab/Placebo Plus Trastuzumab Plus Chemotherapy in Human Epidermal Growth Factor Receptor 2 Positive (HER2+) Advanced Gastric or Gastroesophageal Junction (GEJ) Adenocarcinoma (MK3475-811/KEYNOTE-811). Available online: https:// www.clinicaltrials.gov/ct2/show/NCT03615326

49. Safran H, Winter KA, Wigle DA, et al. Trastuzumab with trimodality treatment for esophageal adenocarcinoma with HER2 overexpression: NRG Oncology/RTOG 1010. J Clin Oncol 2020;38:4500.

50. Abali H, Yalcin S, Onal C, et al. A Study of the Combination of Oxaliplatin, Capecitabine, and Trastuzumab With Chemoradiotherapy in the Adjuvant Setting in Operated Participants With Human Epidermal Growth Factor Receptor-2 Positive (HER2+) Gastric or Gastroesophageal Junction Cancer (TOXAG). J Clin Oncol 2018;36:26.

51. Cunningham D, Allum WH, Stenning SP, et al. Perioperative chemotherapy versus surgery alone for resectable gastroesophageal cancer. $\mathrm{N}$ Engl J Med 2006;355:11-20.

52. Rivera F, Jimenez-Fonseca P, Alfonso PG, et al. NEOHX study: Perioperative treatment with trastuzumab in combination with capecitabine and oxaliplatin (XELOX-T) in patients with HER-2 resectable stomach or esophagogastric junction (EGJ) adenocarcinoma-18 m DFS analysis. J Clin Oncol 2015;33. doi: 10.1200/ jco.2015.33.3_suppl.107.

53. Hofheinz R, Hegewisch-Becker S, Thus-Patience PC, et 
al. HER-FLOT: Trastuzumab in combination with FLOT as perioperative treatment for patients with HER2-positive locally advanced esophagogastric adenocarcinoma: A phase II trial of the AIO Gastric Cancer Study Group. J Clin Oncol 2014;32:4073.

54. Cortes J, Dieras V, Lorenzen S, et al. Trastuzumab emtansine (T-DM1) + capecitabine in HER2-positive metastatic breast cancer $(\mathrm{mBC})$ and HER2-positive locally advanced (LA)/metastatic gastric cancer (mGC): Results from the phase I/randomized phase II TRAXHER2 study. Cancer Res 2018;78:CT096.

55. Shitara K, Bang YJ, Iwasa S, et al. Trastuzumab Deruxtecan in Previously Treated HER2-Positive Gastric Cancer. N Engl J Med 2020;382:2419-30.

56. DS-8201a in HER2-positive Gastric Cancer That Cannot Be Surgically Removed or Has Spread (DESTINYGastric02). Available online: https://clinicaltrials.gov/ct2/ show/NCT04014075

57. Neoadjuvant Study Using Trastuzumab or Trastuzumab With Pertuzumab in Gastric or Gastroesophageal Junction Adenocarcinoma (INNOVATION). Available online: https://clinicaltrials.gov/ct2/show/NCT02205047

58. Holfheinz R, Haag GM, Ettrich TJ, et al. Perioperative trastuzumab and pertuzumab in combination with FLOT versus FLOT alone for HER2-positive resectable esophagogastric adenocarcinoma: Final results of the PETRARCA multicenter randomized phase II trial of the AIO. J Clin Oncol 2020;38:4502.

59. Trial of ZW25 in Patients With Advanced HER2expressing Cancers. Available online: https://clinicaltrials. gov/ct2/show/NCT02892123

60. Combination Margetuximab, INCMGA00012, MGD013, and Chemotherapy Phase 2/3 Trial in HER2+ Gastric/ GEJ Cancer (MAHOGANY). Available online: https:// clinicaltrials.gov/ct2/show/NCT04082364

61. Fuchs CS, Tomasek J, Yong CJ, et al. Ramucirumab monotherapy for previously treated advanced gastric or gastrooesophageal junction adenocarcinoma (REGARD): an international, randomised, multicentre, placebocontrolled, phase 3 trial. Lancet 2014;383:31-9.

62. Wilke H, Muro K, Van Cutsem E, et al. Ramucirumab plus paclitaxel versus placebo plus paclitaxel in patients with previously treated advanced gastric or gastro-oesophageal junction adenocarcinoma (RAINBOW): a double-blind, randomised phase 3 trial. Lancet Oncol 2014;15:1224-35.

63. Pavlakis N, Sjoquist KM, Martin AJ, et al. Regorafenib for the Treatment of Advanced Gastric Cancer (INTEGRATE): A Multinational Placebo-Controlled
Phase II Trial. J Clin Oncol 2016;34:2728-35.

64. Li J, Qin S, Xiong J, et al. Randomized, Double-Blind, Placebo-Controlled Phase III Trial of Apatinib in Patients With Chemotherapy-Refractory Advanced or Metastatic Adenocarcinoma of the Stomach or Gastroesophageal Junction. J Clin Oncol 2016;34:1448-54.

65. Sahin U, Tureci Ö, Manikhas GM, et al. Zolbetuximab combined with EOX as first-line therapy in advanced CLDN18.2+ gastric (G) and gastroesophageal junction (GEJ) adenocarcinoma: updated results from the FAST trial. J Clin Oncol 2019;37:16.

66. Cunningham D, Stenning SP, Smyth EC, et al. Perioperative chemotherapy with or without bevacizumab in operable oesophagogastric adenocarcinoma (UK Medical Research Council ST03): primary analysis results of a multicentre, open-label, randomised phase 2-3 trial. Lancet Oncol 2017;18:357-70.

67. Fuchs CS, Shitara K, Di Bartolomeo M, et al. Ramucirumab with cisplatin and fluoropyrimidine as first-line therapy in patients with metastatic gastric or junctional adenocarcinoma (RAINFALL): a double-blind, randomised, placebo-controlled, phase 3 trial. Lancet Oncol 2019;20:420-35.

68. Sakai D, Boku N, Kodera Y, et al. An intergroup phase III trial of ramucirumab plus irinotecan in third or more line beyond progression after ramucirumab for advanced gastric cancer (RINDBeRG trial). J Clin Oncol 2018;36:4138.

69. Lorenzen S, Thuss-Patience PC, Pauligk C, et al. FOLFIRI plus ramucirumab versus paclitaxel plus ramucirumab as second-line therapy for patients with advanced or metastatic gastroesophageal adenocarcinoma with or without prior docetaxel: Results from the phase II RAMIRIS Study of the AIO. J Clin Oncol 2020;38:4514.

70. FLOT vs. FLOT/Ramucirumab for Perioperative Therapy of Gastric or GEJ Cancer (RAMSES) (RAMSES/ FLOT7). Available online: https://clinicaltrials.gov/ct2/ show/NCT02661971

71. Van Cutsem E, Tabernero J, Lakomy R, et al. Addition of aflibercept to fluorouracil, leucovorin, and irinotecan improves survival in a phase III randomized trial in patients with metastatic colorectal cancer previously treated with an oxaliplatin-based regimen. J Clin Oncol 2012;30:3499-506.

72. Cleary JM, Horick NK, McCleary NJ, et al. FOLFOX plus ziv-aflibercept or placebo in first-line metastatic esophagogastric adenocarcinoma: a double-blind, randomized, multicenter phase 2 trial. Cancer 2019;125:2213-21. 
73. Bang YJ, Kang YK, Kang WK, et al. Phase II study of sunitinib as second-line treatment for advanced gastric cancer. Invest New Drugs 2011;29:1449-58.

74. Moehler M, Mueller A, Hartmann JT, et al. An openlabel, multicentre biomarker-oriented AIO phase II trial of sunitinib for patients with chemo-refractory advanced gastric cancer. Eur J Cancer 2011;47:1511-20.

75. Schmitt JM, Sommers SR, Fisher W, et al. Sunitinib plus paclitaxel in patients with advanced esophageal cancer: a phase II study from the Hoosier Oncology Group. J Thorac Oncol 2012;7:760-3.

76. Yi JH, Lee J, Lee J, et al. Randomised phase II trial of docetaxel and sunitinib in patients with metastatic gastric cancer who were previously treated with fluoropyrimidine and platinum. Br J Cancer 2012;106:1469-74.

77. Sun W, Powell M, O'Dwyer PJ, et al. Phase II study of sorafenib in combination with docetaxel and cisplatin in the treatment of metastatic or advanced gastric and gastroesophageal junction adenocarcinoma: ECOG 5203. J Clin Oncol 2010;28:2947-51.

78. Martin-Richard M, Gallego R, Pericay C, et al. Multicenter phase II study of oxaliplatin and sorafenib in advanced gastric adenocarcinoma after failure of cisplatin and fluoropyrimidine treatment. A GEMCAD study. Invest New Drugs 2013;31:1573-9.

79. A Study of Regorafenib in Refractory Advanced GastroOesophageal Cancer (INTEGRATEII). Available online: https://clinicaltrials.gov/ct2/show/NCT02773524

80. Fukuoka S, Hara H, Takahashi N, et al. Regorafenib Plus Nivolumab in Patients With Advanced Gastric or Colorectal Cancer: An Open-Label, Dose-Escalation, and Dose-Expansion Phase Ib Trial (REGONIVO, EPOC1603). J Clin Oncol 2020;38:2053-61.

81. Regorafenib in Combination With Paclitaxel in Advanced Oesophagogastric Carcinoma (REPEAT). Available online: https://clinicaltrials.gov/ct2/show/NCT02406170

82. Li J, Qin S, Xu J, et al. Apatinib for chemotherapyrefractory advanced metastatic gastric cancer: results from a randomized, placebo-controlled, parallel-arm, phase II trial. J Clin Oncol 2013;31:3219-25.

83. Kang Y, Kang WK, Di Bartolomeo M, et al. Randomized phase III ANGEL study of rivoceranib (apatinib) + best supportive care (BSC) vs placebo + BSC in patients with advanced/metastatic gastric cancer who failed $\geq 2$ prior chemotherapy regimens. Ann Oncol 2019;30:v851-v934.

84. Sahin U, Koslowski M, Dhaene K, et al. Claudin-18 splice variant 2 is a pan-cancer target suitable for therapeutic antibody development. Clin Cancer Res 2008;14:7624-34.

85. A Phase 3 Efficacy, Safety, and Tolerability Study of Zolbetuximab (Experimental Drug) Plus mFOLFOX6 Chemotherapy Compared to Placebo Plus mFOLFOX6 as Treatment for Gastric and Gastroesophageal Junction (GEJ) Cancer (SPOTLIGHT). Available online: https:// ClinicalTrials.gov/show/NCT03504397

86. Trusolino L, Bertotti A, Comoglio PM. MET signaling: principles and functions in development, organ regeneration and cancer. Nat Rev Mol Cell Biol 2010;11:834-48.

87. Iveson T, Donehower RC, Davidenko I, et al. Rilotumumab in combination with epirubicin, cisplatin, and capecitabine as first-line treatment for gastric or oesophagogastric junction adenocarcinoma: an open-label, dose de-escalation phase $1 \mathrm{~b}$ study and a double-blind, randomised phase 2 study. Lancet Oncol 2014;15:1007-18.

88. Lang SA, Gaumann A, Koehl GE, et al. Mammalian target of rapamycin is activated in human gastric cancer and serves as a target for therapy in an experimental model. Int J Cancer 2007;120:1803-10.

89. An JY, Kim KM, Choi MG, et al. Prognostic role of p-mTOR expression in cancer tissues and metastatic lymph nodes in p T2 b gastric cancer. Int J Cancer 2010;126:2904-13.

90. Doi T, Muro K, Boku N, et al. Multicenter phase II study of everolimus in patients with previously treated metastatic gastric cancer. J Clin Oncol 2010;28:1904-10.

91. Murai J, Huang SY, Das BB, et al. Trapping of PARP1 and PARP2 by clinical PARP inhibitors. Cancer Res 2012;72:5588-99.

92. Farmer H, McCabe N, Lord CJ, et al. Targeting the DNA repair defect in BRCA mutant cells as a therapeutic strategy. Nature 2005;434:917-21.

93. Bang, YJ, Im SA, Lee KW, et al. Randomized, DoubleBlind Phase II Trial With Prospective Classification by ATM Protein Level to Evaluate the Efficacy and Tolerability of Olaparib Plus Paclitaxel in Patients With Recurrent or Metastatic Gastric Cancer. J Clin Oncol 2015;33:3858-65.

Cite this article as: Hsu A, Chudasama R, Almhanna K, Raufi A. Targeted therapies for gastroesophageal cancers. Ann Transl Med 2020;8(17):1104. doi: 10.21037/atm-20-3265 